\title{
Optimization models for the use of agricultural aviation when performing chemical works
}

\author{
Abdizhapar Saparbayev ${ }^{1,2, *}$, Aiymzhan Makulova ${ }^{3, * *}$, Assel Kaziyeva ${ }^{4, * * *}$, Zaure Klyshbayeva ${ }^{5, * * * *}$, and Elmira \\ Saparbayeva ${ }^{6, \dagger}$ \\ ${ }^{1}$ Academy Kainar, 7a Satpayev street, Almaty, 050013, Kazakhstan \\ ${ }^{2}$ International Academy of Innovative Technologies, 33 mkr. Samal-2, Almaty, 050059, Kazakhstan \\ ${ }^{3}$ University Narxoz, 55 Zhandosov Str., Almaty, 050035, Kazakhstan \\ ${ }^{4}$ Abai Kazakh National Pedagogical University, 13 Dostyk Ave., Almaty, 050010, Kazakhstan \\ ${ }^{5}$ College of Catering and Service, 12 B. Mailin Str., Nur-Sultan, 010008, Kazakhstan \\ ${ }^{6}$ Mukhtar Auezov South Kazakhstan University, 5 Tauke Khan Ave., Shymkent, 160012, Kazakhstan
}

\begin{abstract}
The article discusses the optimization models for the use of agricultural aviation when performing chemical works. The question of determining the need of vehicles for the maintenance of agricultural work is of great national economic importance. Aviachemical method is firmly established in the technology of cultivation of grain crops. First of all, this is due to the fact that, compared to ground-based methods, the aerochemical method of chemization of agriculture has a number of technical and economic advantages, including a higher quality and uniformity of chemicals, eliminates mechanical damage to crops, and less dependence on the physical and geographical conditions of the area, allows to obtain significant savings of chemicals and fuel, reduces the time of work.
\end{abstract}

\section{Introduction}

Of particular importance is the use of aviation technology in the conditions of intensification of grain production. With the help of aviation equipment, mineral fertilizers are applied, aviation-chemical control of pests and diseases of field plants is carried out, weed vegetation is destroyed in grain crops, defoliation, desiccation of plants and a number of other works are carried out. Currently, with the help of aviation, more than a third of the chemical work in the grain industry is performed and more than 20 million hectares of land are processed annually [1].

Such a diverse use of aviation technology in grain farming is primarily due to the fact that the implementation of aviation chemical works on technical, economic and economic efficiency is not inferior to the ground-based method, and for a number of indicators, such as productivity, reduced labor costs, the possibility of processing by wet soil without over compaction and destruction of its structure, the exclusion of mechanical damage exceeds it. In addition, the use of aviation technology allows freeing up human resources: over a year, labor savings amount to more than 15 million man-days and about 6 million tractor shifts. According to scientific studies, on average, the aviation method of processing crops of grain crops, as compared with the ground, gives an additional yield increase

\footnotetext{
*e-mail: sad171@mail.ru

**e-mail: aiymzhan.makulova@narxoz.kz

***e-mail: assel01@mail.ru

****e-mail: zaure1975.75@mail.ru

†e-mail: saparbaeva_e@list.ru
}

of up to $2 \mathrm{c} / \mathrm{ha}$. Additional net income from the use of aviation in grain farming exceeds 3 billion tenge [2].

\section{Material and methods}

The use of an aircraft fleet (AF) when performing chemical works in the territory of the republic is characterized, on the one hand, by a large variety of types of these works, and on the other hand, by the uneven intensity of their performance in time and territory. In the transition from one type of work to another, both the productivity of the technical equipment that performs these works and the cost of their implementation changes. Therefore, with optimal distribution of the aircraft fleet at the aviation chemical works (AChW), conditions are created for the fullest possible satisfaction of applications for aviation service due to more intensive use of the aircraft.

AChW performance with deadlines, established by agrotechnology, can dramatically reduce the effect of the activities carried out, and sometimes completely unacceptable. Compressed and tightly fixed deadlines for conducting an emergency management dictate a particular area for a certain period of time, the need for such an aircraft fleet, which significantly exceeds the capacity of an airline located in a given area. "Peak" periods in different regions of the republic may partially or completely not coincide in time, which is explained by differences in climatic conditions and in "peak" periods in different regions of the republic may partially or completely not coincide in time, which is explained by differences in climatic conditions 
and in "peak" periods in different regions of the republic may partially or completely not coincide in time, which is explained by differences in climatic conditions and in structure of cultivated crops and land. Such a discrepancy in time of "peak" periods in various areas creates the necessary prerequisites for the temporary transfer of part of the aircraft fleet from home areas to other areas currently in need, i.e. for maneuvering fleet in the Republic.

The heterogeneity of the structure of cultivated crops and land in areas, mainly due to differences in soil and climatic conditions, is the main reason for the discrepancy between the "peaks" of AChW in these areas. Such a discrepancy in time of the periods of maximally intensive use of aircraft parks allows defining a simple logic scheme for redistributing the aircraft fleet between them at certain time intervals. Such redistribution, i.e. maneuvering allows you to perform a much larger amount of $\mathrm{AChW}$ at the agrotechnical periods most favorable for the regions without increasing the fleet in the aviation enterprises of each of them.

\section{Results and discussion}

To solve the problem of the optimal redistribution of agricultural aviation between areas (temporary secondment), taking into account the mismatch of their "peak" periods, the usual deterministic linear programming model can be used. Calculations on such a model provide a practically acceptable solution to the maneuvering problem, especially if it is solved as an operational management task, i.e. for a short time interval (decade, month). However, with an increase in the planning interval (quarter, year), apparently, the accuracy and, consequently, the practical suitability of calculations for the deterministic model can deteriorate sharply due to the fact that the influence of the random factor is not taken into account in such a model. So, in fact, the "peak" period in a region may not only not coincide in time with similar periods in other regions, but also under the influence of weather conditions can significantly mix with respect to the time interval in which its manifestation was previously (predicted). Therefore, a more accurate solution to the problem of using agricultural aviation in the performance of chemical work, taking into account the random factor, can be obtained by solving it in a stochastic formulation.

When constructing and studying mathematical models of the use of the fleet of aircraft for the performance of maintenance and repair, the following should be considered:

- the priority of the performance of the aircraft security personnel, for which the serviced territory is assigned;

- multipurpose use of aircraft due to the diversity of the types of work performed;

- the discrepancy of the situation during the year of the peaks of the performance of the AChW in different areas of the service area;

- the implementation of labor-intensive regulations is carried out at home enterprises.
Taking into account the above features, a mathematical model of optimal maneuvering of aircraft fleet on AChW in the context of current planning can be formulated as a multi-index production-transport task of resource allocation: find such a plan of maneuvering aircraft with which the expected amount of maintenance is performed with a minimum of total reduced costs.

It is necessary to find a solution that minimizes the reduced total costs $[1,3,4]$ :

$$
\sum_{i=1}^{m} \sum_{j=1}^{n} \sum_{l=1}^{L} \sum_{r=1}^{R} \sum_{t=1}^{T} C_{i j e r} x_{i j e r t}
$$

under restrictions on the amount of work performed

$$
\sum_{i=1}^{m} \sum_{l=1}^{L} P_{i j r} x_{i j l r t} \geq d_{j r t},
$$

for the number of serviceable aircraft

$$
\sum_{i=1}^{n} \sum_{r=1}^{R} x_{i j l r t} \geq a_{i l t}, i=\overline{1, m}, l=\overline{1, L}, t=\overline{1, T}
$$

for consumption of fuels and lubricants

$$
\sum_{i=1}^{m} \sum_{j=1}^{n} \sum_{l=1}^{L} \sum_{r=1}^{R} q_{i} x_{i j l r t} \leq Q_{t}, t=\overline{1, T},
$$

with the natural requirement of nonnegativity of variables

$$
\begin{aligned}
& x_{i j l r t} \geq 0, i=\overline{1, m} ; \\
& j=\overline{1, n} ; r=\overline{1, R} ; l=\overline{1, L} ; t=\overline{1, T} ;
\end{aligned}
$$

where $i-$ an index of type AF, $i=\overline{1, m}$;

$j-$ an index of type AChW, $j=\overline{1, n}$;

$l$ - airline index in which the aircraft is based AF, $l=\overline{1, L}$;

$r$-index of the area (region) of the AChW, $r=\overline{1, R}$;

$t-$ the index of the subperiod, $t=\overline{1, T}$;

$d_{i r t}$ - the volume of chemical works of the $j$-th species in the $r$-th region, which must be performed in the subperiod $t$, ha;

$a_{i l t}$ is the number of serviceable aircraft of the $i$-th type at enterprises $l$ in the sub-period $t$;

$C_{i j l r}$ - unit costs of the enterprise $l$ for the execution of works of the $j$-th type by the $i$-th type of aircraft in the region of $r$, tenge;

$P_{i j r}$ - specific productivity of the $i$-th aircraft type, performing the $j$-th type of work in the $r$-th region, ha/h;

$q_{i}$ - specific fuel consumption of the $i$-th aircraft type, $\mathrm{kg} / \mathrm{h}$;

$Q_{t}-$ disposable amount of fuel and lubricants in the subperiod $t, \mathrm{t}$;

$X_{i j l r t}$ - number of operated aircraft of the $i$-th type assigned to the aviation enterprise $l$ in the $j$-th type of work in the $r$-th region in the sub-period $t$.

The task of maneuvering the fleet of aircraft on the AChW in the formulation (1) - (5) belongs to the class of linear programming problems. When solving the problem (1) - (5) by conventional linear programming methods, considerable difficulties arise associated with the high 
dimensionality of the problem (the number of variables is $m n l R T=3 \cdot 4 \cdot 21 \cdot 28 \cdot 12$; the number of constraints is $n R T+m L T+T=4 \cdot 12 \cdot 28+3 \cdot 12 \cdot 21+12)$. Over the past two decades, many characteristic structures have been identified and a large number of algorithms have been created to solve them [5-7]. Two main approaches to solving problems of large dimensionality were defined: direct methods and methods based on ideas of decomposition (decomposition).

In the first case, for solving problems of a special type, general methods of mathematical programming are used. In this case, the main computational difficulties associated with the storage of large amounts of information and operations on them. Large sparse matrices (matrices having a small percentage of nonzero elements) are usually stored in computer equipment in packaged form, i.e. only nonzero elements are stored along with the necessary information about their position in the matrix. For example, when using the simplex method for solving linear problems of large dimension, the main computational difficulties are associated with the inversion of the basis matrix [8]. Therefore, when solving a large dimension problem, it is advisable to use a modified simplex method or one of the modifications of the generalized gradient descent method. A feature of the modified simplex method is the representation of the inverse matrix of the conditions of the problem in a multiplicative form, which provides a number of significant computational advantages. Most modern mathematical programming packages use the modified simplex method. If the matrix of conditions of the problem has a special structure, but the necessary calculations can be performed using a matrix of a lower dimension. Such an approach, for example, is implemented when applying the methods of taking into account bilateral restrictions or the method of reducing the basis to a triangular shape. Most modern mathematical programming packages use the modified simplex method. If the matrix of conditions of the problem has a special structure, but the necessary calculations can be performed using a matrix of a lower dimension. Such an approach, for example, is implemented when applying the methods of taking into account bilateral restrictions or the method of reducing the basis to a triangular shape. Most modern mathematical programming packages use the modified simplex method [9, chapter 3]. If the matrix of conditions of the problem has a special structure, but the necessary calculations can be performed using a matrix of a lower dimension. Such an approach, for example, is implemented when applying the methods of taking into account bilateral restrictions or the method of reducing the basis to a triangular shape.

The methods belonging to the second class are based on decomposing the system into subsystems of a lower dimension. Usually, the first and second level subsystems are distinguished in such a way that the second level subsystems determine the corresponding changes in the first level subsystems. The task of the second level is to coordinate the functioning of the elements of the first level in order to obtain a common solution to the original problem.

Two main decomposition approaches were put forward in the study of large linear programming problems [10-
12]. The principles of Danzig-Wolfe entailed a numerous stream of works developing central ideas in various directions. If in the original version the solution of the coordinating problem is based on the method of improving the plan in linear programming, in subsequent works they proceeded from the method of simultaneous solution of the direct and dual problems, the generalized gradient descent, the use of modified Lagrange functions and other procedures.

Analysis of the existing use of aircraft and the seasonality of the AChW, the monthly maintenance of accounting and reporting documentation for airlines shows that a monthly maneuvering scheme is justified, with the aircraft returning to the base enterprise by the beginning of each month. Repeated flights in the adjacent two months, possible in a common solution for the entire planned period, can be eliminated by their subsequent analysis. Therefore, the task (1) - (5) will be solved for each "peak" month separately.

When implementing the task (1) - (5) it is advisable to consider the following.

1. In practice, it is necessary to perform calculations on the model for several objective functions.

2. In actual calculations, indexes of types of aircraft, types of maintenance organizations, airlines and serviced areas do not take all values, but only their various subsets. For example, for each airline, only a certain subset of the serviced areas can be defined, in which a certain subset of the types of maintenance should be performed. To describe this possibility, it is advisable to use in the model not an enumeration of index values from minimum to maximum, but an indication of its subset.

3. There is a need to solve the problem with a restriction on the fuel resource as a whole in terms of management, and sometimes even in the context of individual airlines.

4. The statement of the problem (1) - (5) is rigid, i.e. a situation of no valid solution is possible. However, this situation can be interpreted economically (as a deficit of the fleet of aircraft or an overestimated amount of work). Therefore, it is also of interest to the planner to analyze an admissible solution. In order to ensure the possibility of such an analysis, it is better to use instead of setting (1) (5) a non-rigid statement with discrepancies in constraints that may become critical in the calculation process. But to match the solutions on the model (1) - (5) and the proposals of the following models by us, the sum of the residuals with the latter must be entered into the objective function with the corresponding penalty coefficient (large enough - positive) when solving the problem at minimum or very small (large negative) - to the maximum.

The need to take these four points into account caused the transition in the MANEVR dialogue complex from us- 
ing the model $(1)-(5)$ in the next model in the so-called technological formulation.

\section{Calculated ratios}

1. The calculation of the total costs associated with maneuvering the aircraft and the execution of works:

$$
\sum_{l=1}^{L} \sum_{r \in R_{l}} \sum_{j \in N_{r}} \sum_{i \in M_{l r j}} \tilde{c}_{r}^{i j} x_{e r}^{i j}-y_{1}=0
$$

2. The calculation of the total value (tariffs) of work performed:

$$
\sum_{l=1}^{L} \sum_{r \in R_{1}} \sum_{j \in M_{i r j}} \tilde{S}_{r}^{i j} x_{e r}^{i j}-y_{2}=0
$$

3. The calculation of the total amount of outstanding work:

$$
\sum_{r \in R_{e}} \sum_{j \in N_{r}} V_{i}^{j}-y_{0}=0
$$

\section{Target functions}

1. The minimization of the total cost of the AChW:

$$
\mu y_{0}+\epsilon y_{1} \rightarrow \min
$$

2. Maximizing profits from the performance of the AChW:

$$
\epsilon\left(y_{2}+y_{1}\right)-\mu y_{0} \rightarrow \max
$$

III. The main resource and technological limitations

1. Restrictions on the fleet of aircraft in the airline (in terms of aircraft days):

$$
\sum_{r \in R_{e}} \sum_{j \in N_{r}} x_{e r}^{i j} \leq a_{e}^{i}, I=\overline{1, L} ; i \in M_{1}
$$

2. Restrictions on the volume of work performed:

$$
\sum_{l=1}^{L} \sum_{i \in M_{l r j}} P_{r}^{j i} x_{l r}^{i j}+V_{r}^{j}=d_{r}^{j}, r \in R_{1} ; j \in N_{r}
$$

3. Restrictions on fuel resources:

$$
\sum_{r \in R_{l}} \sum_{j \in N_{r}} \sum_{i \in M_{k}} q_{i}^{k} x_{l r}^{i j} \leq Q_{l}^{k}, l=\overline{1, L} ; k=1,2 .
$$

or alternative

$$
\sum_{L}^{l=1} \sum_{r \in R_{1}} \sum_{j \in N_{r}} \sum_{i \in M_{l}} q^{k} x_{l r}^{i j} \leq Q^{k}, k=1,2 .
$$

IV. Nonnegativity conditions $x_{l r} \leq 0, y_{0} \leq 0, Y_{1} \leq$ $0, y_{2} \leq 0$

$$
V_{r} \leq 0, i \in M_{1}, j \in N_{r}, l=\overline{1, L} ; r \in R_{1}
$$

The designations of the model (6) - (15) are similar to the designations of the model $(1)-(5)$ with the exception of the following: $k$ - fuel type index; $R_{1}-$ a subset of the serviced areas allowed for the 1st airline; $N_{r}$ - a subset of the work performed in the $r$-th region; $M_{l r j}$ - a subset of aircraft types available in the $l$-st airline; $\tilde{C}_{l r}^{i j}-$ complex costs (specific) for the execution of works, taking into account maneuvering,

$$
\tilde{C}_{e r}^{i j}=C_{i e}^{l . c h} \cdot h_{e}^{i}+C_{i e}^{l . c h .} \cdot \frac{2 L e r}{V_{i}} \frac{h_{e}^{i}}{\left(T_{i}-\frac{2 L e r}{V_{i}}\right)}
$$

where $C_{i l}^{\text {l.ch. }}$ - cost of flight hours;

Ler - distances between airlines and serviced areas, $\mathrm{km}$;

$V_{i}$ - average ground speed;

$T_{I}$ - time limit (monthly flying hours);

$\tilde{S}_{r}^{i j}$ - cost of works (calculated as a function of summation of tariff rates for a single treatment of 1 ha, established depending on the type of aircraft, type of work, the consumption rate of the substance and the rut length of the cultivated area and tariff rates per 1 hectare for approaching the aircraft from the aerodrome to the cultivated area depending on the type of aircraft and the approach distance);

$a_{1}$ - the fleet of aircraft (in terms of aircraft days);

$\mu$ and $\epsilon-$ weighting coefficients which establish the correct priorities for the objective function component $\left(\mu \sim 10^{3}\right.$ and $\left.\epsilon \sim 10^{-2}\right)$;

$x_{l r}^{i j}$ - amount of work j-th type AChWs Taken 1-th airlines of a r-th region via i-th type aircraft;

$V_{r}$ - the volume of outstanding work in each area;

$A t_{0}$ - the amount of backlog;

$I_{1}$ is the sum of the costs;

$A t_{2}$ - the total cost of the work.

In the dialogue complex "MANEVR" coefficients $\tilde{C}_{l r}^{i j}$ and $\tilde{S}_{r}^{i j}$ and models (6) - (15) are calculated by a specially developed program in the on-line mode.

So far, only the movement of the aircraft has been considered. But when performing chemical work, it is also practiced to move flight technical crews while maneuvering aircraft in the serviced territory of the republic. The relocation of the flight technical staff will make it possible to rationally combine the labor and material resources of airlines to obtain high economic indicators.

It is necessary to find such a maneuvering plan, in which the declared amounts of AChW will be performed with the maximum profit for the given resource constraints. Mathematically, this problem is written as follows.

Maximize

$$
\sum_{i=1}^{m} \sum_{j=1}^{n} \sum_{l=1}^{L} \sum_{r=1}^{R} \sum_{t=1}^{T}\left(\tau_{i j} P_{i j r}-C_{i j l}\right) X_{i j l r t},
$$

under restrictions on the amount of chemical work

$$
\sum_{i=1}^{m} \sum_{l=1}^{L} P_{i j r} X_{i j l r t} \leq d_{j r t}, j=\overline{1, n}, l=\overline{(1, L) ; t}=\overline{1, T}
$$


for the number of serviceable aircraft

$$
\sum_{j=1}^{n} \sum_{r=1}^{R} X_{i j l r t} \leq a_{i l t}, i=\overline{1, m}, l=\overline{(1, L)} ; t=\overline{1, T}
$$

on the number of crews

$$
\sum_{j=1}^{n} \sum_{r=1}^{R} F_{i} X_{i j l r t} \leq G_{i l t}, i=\overline{1, m}, l=\overline{(1, L) ; t}=\overline{1, T}
$$

on the number of aircraft technicians

$$
\sum_{j=1}^{n} \sum_{r=1}^{R} \alpha_{i} X_{i j l r t} \leq V_{i l t}, i=\overline{1, m}, l=\overline{(1, L) ; t}=\overline{1, T}
$$

for consumption of fuels and lubricants

$$
\sum_{j=1}^{n} \sum_{r=1}^{R} \sum_{t=1}^{T} q_{i j} X_{i j l r t} \leq Q_{l t}, l=\overline{1, L}, l=\overline{1, L} ;
$$

on the power of aviation technical bases

$$
\sum_{j=1}^{n} \sum_{r=1}^{R} \sum_{t=1}^{T} \lambda_{i} X_{i j l r t} \leq M_{l t}, l=\overline{1, m}, l=\overline{1, L} ;
$$

on variables

$$
X_{i j l r t} \geq 0, i=\overline{1, m} ; j=\overline{1, n} ; l=\overline{1, L} ; r=\overline{1, R} ; t=\overline{1, T} .
$$

The indices and most of the designations of the model (16) - (23) coincide with the indices and designations adopted in (1) - (5). In the model (16) - (23) the following notation is added:

$\tau_{i j}$-tariff for processing $i$ ha of the $j$-th type of AChW, tenge;

$G_{i l t}$ is the available number of crews of aircraft of type $i$ at airline $I$ in sub-period $t$;

$\delta_{i}$ - the number of people in the flight crew of the $i$-th type aircraft;

$\alpha_{i}$ - number of members of the technical personnel of the aircraft of the $i$-th type;

$V_{i l t}$ is the available number of aircraft technicians of the $i$-th type aircraft at airline $l$ in sub-period $t$;

$\lambda_{i}$ - the specific volume of maintenance of the $i$-th type aircraft, $\mathrm{g}$;

$M_{i l}$ is the total available capacity of aviation technical bases of airlines $l$ for servicing aircraft of the $i$-th type, h.

Information support of the task is based on data on aircraft parameters, technological features of the performance of certain types of $\mathrm{AChW}$, flight distance to the work site, etc.

As a result of solving the problems of linear programming (16) - (23), we will get not only the optimal maneuvering scheme $\left\{x_{i j l r t}^{*}\right\}$, but also a number of important matters for the user of technical and economic service indicators. So, thanks to the obtained maneuvering scheme, the optimal fuel reserves at the aviation enterprises, the deficit and excess of the fleet, the expedient redeployment of the fleet, etc. are easily determined. Experimental calculations using the model (6) - (15) were performed on real data from the Aviation Administration Department in the national economy of the Kazakh Civil Aviation Authority using the developed and specialized dialogue complex "MANEVR" [2]. Consider the specific results of calculations of optimal plans, made using the complex "MANEVR" for the "peak" period we give quantitative estimates of their effectiveness.

The results of the optimization calculations showed that the share of work performed by the aviation enterprises of the Kazakh Civil Aviation Authority due to maneuvering in the total volume of the $\mathrm{AChW}$ is rather high and amounts to $36.6 \%$. Out of 18 airlines that service 19 regions of the republic, 17 airlines perform the work on the territory of other regions (maneuver) according to the optimal plan, and only the Atyrau airline carries out AChW exclusively in the area of its deployment. The amount of work performed by maneuvering increases significantly even as compared with the optimal plan calculated using fixed connections $(36.6 \%$ vs. $31.7 \%$ in terms of fixed links). This allows us to conclude that, at least for the "peak" period, maneuvering the fleet is an important factor in increasing the efficiency of the performance of maintenance of operational personnel in the republic. Expanding maneuvering capabilities by taking into account the full links between airlines when servicing areas allows you to calculate more effective plan options, both in terms of the costs of performing maintenance, and the net cost of maneuvering. For example, for each month of a "peak" period, the costs of performing an $\mathrm{AChW}$ in the optimal plan calculated on full links are, on average, 2-5\% lower than similar costs in the optimal plan calculated on fixed links. A similar comparison of the net cost of maneuvering results in 10-50\% cost reduction. The effectiveness of the optimization calculations of the maneuvering plan is also visible in terms of its level of balance.

Complex "MANEVR" allows you to calculate the profitability ratios carried out $\mathrm{AChW}$ as a whole by default, and in terms of airline. A comparison of the achieved level of profitability in the basic calculation (for fixed connections) with the optimal plan (for full connections) shows that with optimal maneuvering of the fleet during the "peak" period, the profitability of management as a whole may increase by at least $10-20 \%$, while an increase in the share of highly profitable airlines (from $80 \%$ profitability and above) on average $60 \%$.

Since the optimization calculations were carried out according to two criteria - minimum costs and maximum profits, it should be clarified that the highest profitability rates of airlines are achieved in terms of maximum profits. Moreover, this criterion provides the most narrow range of differences in the level of profitability of AChW airlines. Comparison of calculations for the minimum cost and maximum profit showed that in the first case, the sun than in the second case.

In the previously implemented methodology, optimization calculations using the model (6) - (15) using the MANEVR dialogue complex were also carried out on real data of the Ukrainian and Kazakh Civil Aviation Authorities (GA) for 2010-2019. 
Kazakhstan is one of the leading republics in the use of aviation in the grain industry. The Kazakh Civil Aviation Authority annually handles more than 12 million hectares of land, or about $20 \%$ of the total agricultural inventory performed in the CIS. Of these, fertilizer application accounts for 978.5 thousand hectares, plant protection from pests, diseases and weeds - 5629.2 thousand hectares, defoliation and desiccation of grain crops -223.6 thousand hectares.

Currently, the AChWs performed by the Kazakh Department of Civil Aviation comprise several dozen species. Each type is characterized by the purpose, terms, conditions of performance.

On the territory of the republic in its various areas there are 18 airlines. Each of them has a certain fleet of one or more types of aircraft, designed to perform AChW. All types of aircraft are sufficiently universal, i.e. can be used to perform the entire spectrum of basic AChW. The main types of aircraft that are available in airline control companies include the An-2 aircraft and the Mi-2 and Ka-26 helicopters.

An-2 airplanes are more economical with the application of mineral fertilizers and with similar labor-intensive works. Therefore, they remain the main aviation technology for the production of chemical works in the grain industry. With their help, more than $90 \%$ of the total volume of AChW is performed annually. Recently, the possibility of mass production of An-3 aircraft, which are a deep modification of the An-2 aircraft, is being studied.

The use of Mi-2 and Ka-26 helicopters provides in some cases higher efficiency. However, the cost of work is also increasing, since helicopters are a relatively expensive vehicle.

Out of 18 airlines that serve 19 regions of the republic, 17 airlines, according to the optimal plan, perform work in the territory of other regions (maneuver) and in the area of their deployment. From table 1 it is clear that the largest amount of aviation work is carried out to combat weeds and pests and diseases.

The share of work performed by airlines due to maneuvering, to some extent, characterizes the efficiency (rationality) of locating the airlines themselves in the territory of the republic. Aviation enterprises, in which the percentage of work performed by maneuvering is high (50-80\%), should be considered, apparently, as irrationally located (in the part of agricultural aviation), since they perform most of the work in other areas. And to perform work on their territory, even in the "peak" period, they have an excess of Armed Forces. Another group of airlines should include those that practically do not perform work in other areas. The fleet of these airlines is usually not enough to carry out work in their field during the entire "peak" period. According to the results of optimization calculations, such airlines should primarily include Burunday and Kyzylorda. The remaining airlines form a group with an average percentage of the amount of work performed by maneuvering. These enterprises, in our opinion, are most rationally located and optimal in size for the fleet of agricultural use. Their fleet provides more than half of the work in their "own" area on their own, and maneuvering
Table 1. Distribution of AChW between enterprises of the Kazakh State Aviation Administration according to the optimal maneuvering plan in the "peak" period

\begin{tabular}{lllll}
\hline Aviation & \multicolumn{4}{c}{ Processed, thousand hectares } \\
\cline { 2 - 5 } enterprise & $\begin{array}{l}\text { Mineral Weed } \\
\text { fertil- } \\
\text { izers }\end{array}$ & $\begin{array}{l}\text { Pen- } \\
\text { trol }\end{array}$ & $\begin{array}{l}\text { disease } \\
\text { control }\end{array}$ & $\begin{array}{l}\text { Defoliation } \\
\text { and des- } \\
\text { iccation }\end{array}$ \\
\hline Burunday & 21.2 & 21.7 & 8.3 & - \\
West Kaza- & 30.0 & 97.9 & 155.0 & - \\
khstan & & & & \\
Aktobe & 4.5 & 175.6 & 66.9 & - \\
Karaganda & 52.8 & 160.6 & 174.4 & - \\
Kostanay & 69.0 & 448.0 & 262.3 & - \\
Atyrau & - & - & - & - \\
East Kaza- & 3.5 & 84.8 & 53.3 & 7.8 \\
khstan & & & & \\
Turkestan & 281.6 & 256.8 & 154.2 & 35.0 \\
Zhambyl & 90.2 & 223.1 & 145.5 & 39.0 \\
Akmola & 100.6 & 551.4 & 255.2 & 57.7 \\
Semipalatinsk & 51.6 & 96.3 & 201.1 & 4.0 \\
Kokshetau & 45.7 & 262.1 & 168.4 & 18.3 \\
Pavlodar & 4.9 & 87.8 & 498.1 & 24.8 \\
North Kaza- & 29.7 & 193.1 & 71.5 & - \\
khstan & & & & \\
Kzyl-Orda & 50.3 & 15.8 & 0.7 & 35.3 \\
Taldykorgan & 96.2 & 79.6 & 129.4 & - \\
Turgai & 52.7 & 224.3 & 240.0 & - \\
Zhezkazgan & - & 11.4 & 54.6 & - \\
\hline Total man- & 978.5 & 2990.3 & 2638.9 & 223.5 \\
agement & & & & \\
\hline & & & & \\
\hline
\end{tabular}

avoids the irrational use of aircraft in certain months with lower volumes of declared work.

Optimization calculations also make it possible to characterize the level of specialization of airlines in the performance of various maintenance companies and to evaluate the effectiveness (rationality) of their deployment in the republic at the present time.

\section{Conclusion}

The analysis of the calculations performed by the Kazakh State Aviation Administration show the high efficiency of using optimization methods and models for solving the problem of using agricultural aviation in the performance of chemical work. And the practical value of the optimization approach to solving this problem is directly provided by the territorial administration of the GA fully provided by the calculation technology implemented in the MANEVR dialogue complex.

\section{References}

[1] A. Saparbayev, Transport logistics in grain processing (Fortuna Polygraph LLP, 2019)

[2] A. Iztaev, T. Kulazhanov, A. Saparbayev, Innovative technologies and logistics of processing businesses in AIC (Fortuna Polygraph LLP, 2019) 
[3] A. Bakaev, V. Revenko, V. Gritsenko, Information technology in transport, Vol. 3. Water transport (Naukova dumka, Kyiv, 2009)

[4] E. Vasilyeva, V. Levit, V. Livshits, Nonlinear transport problems on networks (Finansy i statistika, Moscow, 1981)

[5] E. Krylatykh, A. Strokov, Food Security of Russia and the World: Theory and Methodology of Research, Analysis of Provision, Opportunities and Threats (Infra-M, 2019)

[6] A. Ganicheva, Mathematical methods and models in agroindustrial complex (Tver State Agricultural Academy, Tver, 2019)

[7] A. Makulova, Model assessment of agricultural production management (Fortuna Polygraph LLP, 2019)
[8] L. Lesdon, Optimization of large systems (Nauka, Moscow, 1975)

[9] H.A. Taha, Operations Research: An Introduction, 10th edn. (Pearson, 2017)

[10] G.B. Dantzig, M.N. Thapa, Linear programming 2: theory and extensions (Springer Science \& Business Media, 2006)

[11] M.R. Bartolacci, L.J. LeBlanc, Y. Kayikci, T.A. Grossman, Journal of Business Logistics 33, 118 (2012)

[12] M. Savelsbergh, J.H. Song, Computers \& Operations Research 35, 2266 (2008), part Special Issue: Includes selected papers presented at the ECCO'04 European Conference on combinatorial Optimization 